\title{
Relation between visceral fat and carotid intimal media thickness in Mexican postmenopausal women: a preliminary report
}

\author{
Sebastián Carranza-Lira' ${ }^{1}$, Yessica Mireya Moreno Azpilcueta ${ }^{2}$, Sergio Rosales Ortiz ${ }^{3}$
}

\begin{abstract}
${ }^{1}$ Chairman of the Research Division in Health, UMAE Gynecology and Obstetrics Hospital "Luis Castelazo Ayala", Social Security Mexican Institute, Mexico DF, Mexico

${ }^{2}$ Obstetrics and Gynecology Resident, UMAE Gynecology and Obstetrics Hospital “Luis Castelazo Ayala”, Social Security Mexican Institute, Mexico DF, Mexico

${ }^{3}$ Ultrasound Service Staff, UMAE Gynecology and Obstetrics Hospital “Luis Castelazo Ayala”, Social Security Mexican Institute, Mexico DF, Mexico
\end{abstract}

\begin{abstract}
Aim of the study: To investigate the relationship between visceral fat and carotid IMT (intima media thickness) in Mexican postmenopausal women.

Material and methods: In 71 postmenopausal women divided in two groups: group 1, IMT >1 mm and group 2, IMT $\leq 1 \mathrm{~mm}$, blood pressure, body mass index (BMI), waist hip ratio (WHR), visceral and subcutaneous fats and carotid IMT were analyzed. Descriptive statistics were used and the comparison among those with abnormal and normal IMT was carried out using Mann-Whitney $U$ test; also Spearman's correlation analysis was done.

Results: When comparing group $1(n=9,12.7 \%)$ with group $2(n=62,87.3 \%)$, it was found that the subcutaneous fat, visceral fat and systolic blood pressure were significantly greater in group $1(p<0.018, p<0.001$ and $p<0.006$, respectively), and also in this group there was a correlation between $\mathrm{BMI}$ and subcutaneous fat $(\rho=0.686, p<0.041)$ and between visceral fat and the systolic blood pressure $(\rho=0.712, p<0.031)$. In group 2 , there was a correlation between IMT and diastolic blood pressure $(\rho=0.251, p<0.049)$.

Conclusion: Subcutaneous and visceral fat have an unfavorable effect in the carotid IMT and in blood pressure.
\end{abstract}

Key words: visceral fat, subcutaneous fat, intima media thickness, postmenopause, carotid, blood pressure.

\section{Introduction}

Since life expectancy has increased, it is expected that in 2030 there will be at least 10 million women in postmenopause [1].

Atherosclerotic cardiovascular disease is the main cause of death among postmenopausal women, probably due to the hormonal changes. A woman, at 50 years of age has a $46 \%$ risk of suffering from coronary artery disease and $31 \%$ risk of dying due to that condition, which is greater than the possibility of death due to cancer or hip fracture [2]. Atherosclerosis is a systemic disease that is responsible for most of the cardiovascular events and strokes and the intima media thickness (IMT) is increased in patients with atherosclerosis [3].

The IMT measured by ultrasound has been validated by official medical organizations [4]. In a recent meta-analysis which comprised 37,000 individuals, it was concluded that a $0.1 \mathrm{~mm}$ increase in IMT increases the risk of myocardial infarction by $10 \%$ to $15 \%$ and that of stroke by $13 \%$ to $18 \%$ [4].

The European Society of Hypertension and the European Cardiology Society guidelines for arterial hypertension management recommend the mensuration of IMT in high risk patients, also the American Heart Association and the American College of Cardiology supported its use in patients with an intermediate risk, if their risk classification was not satisfactory $[5,6]$.

Obesity prevalence increases from premenopause to postmenopause. It is known that overweight and obesity are important health problems associated with metabolic dysfunctions and cardiovascular disease. Also, visceral fat accumulation is associated with insulin resistance, type 2 diabetes and it is an important part of metabolic syndrome [7]. Abdominal fat accumulation has a central role in the pathogenesis of cardiovascular 
disease in obese subjects; also visceral fat is associated with a greater risk of cardiovascular disease. However, some studies have suggested a possible beneficial role of subcutaneous fat $[7,8]$. Anthropometric measurements such as the waist perimeter that is a marker of central obesity, are useful in the clinical practice due to their convenience and non-invasiveness. For abdominal fat evaluation, CAT-scan [9, 10] as well as abdominal ultrasound [11] have been used.

Carotid atherosclerosis is associated with visceral fat accumulation [7], but in Mexican population there are no studies to this respect.

So the objective of the study was to investigate the relationship between visceral fat and carotid IMT in Mexican postmenopausal women.

\section{Material and methods}

Postmenopausal women (12 months or more since menopause) that attended the external consultation of the UMAE for gynecological conditions were studied. Those that had received hormone therapy were not included. In all of them, a clinical history was carried out investigating family history of cardiovascular disease, personal history of type 2 diabetes mellitus and cardiovascular disease. They were interrogated about smoking habits, alcohol and drug consumption, and also the frequency of exercise and how they considered their alimentary habits was investigated. Age at menarche (years), age at menopause (years), pregnancies and childbirths were asked about. Weight $(\mathrm{kg})$, height $(\mathrm{m})$, waist perimeter $(\mathrm{cm})$ and hip perimeter $(\mathrm{cm})$ were measured. The body mass index (BMI, weight/height ${ }^{2}$ ) and waist hip ratio (WHR, waist perimeter/hip perimeter) were calculated considering a lower level fat distribution for $W H R \leq 0.85$ and upper level fat distribution for WHR $>0.85$. The systolic and diastolic blood pressure (BP, $\mathrm{mmHg}$ ) were also measured.

Afterwards an abdominal ultrasound was carried out with a Voluson 730 Pro equipment (General Electric Healthcare, Austria GM GH) a $3.5 \mathrm{MHz}$ probe was used to measure subcutaneous and visceral fat (intrabdominal), for this the transducer was placed in the half line, $1 \mathrm{~cm}$ below the umbilical scar. Subcutaneous fat was considered as the distance between skin and the external face of the rectus abdominis muscle $(\mathrm{cm})$ and the visceral fat was considered as the distance $(\mathrm{cm})$ between the internal face of the rectus abdominis muscle and the anterior aorta wall [11]. After that, the carotid IMT was measured by ultrasound with a $5 \mathrm{MHz}$ probe, locating the carotid artery at $1 \mathrm{~cm}$ to its bifurcation, the image was maximized visualizing the lumen diameter and measuring the distance between the first and the second echogenic line. The greater value was used for the analysis. Carotid IMT was considered to be normal when it was $\leq 1 \mathrm{~mm}$ and abnormal when $>1 \mathrm{~mm}$ [4].
For sample size calculation, the Epi Info 7 program (Centers for Disease Control and Prevention, Atlanta, GA) was used and a 700 women universe was considered, with an esteemed frequency of disease of $12 \%$, with a confidence level of $90 \%$ and a worse expected result of $18 \%$, obtaining a sample size of 71 [12].

Statistical analysis was done with the SPSS program (IBM SPSS Statistics for Windows, Version 20.0. Armonk, NY: IBM Corp.). Descriptive statistics were used, the comparison among those with normal and abnormal IMT, was done using Mann-Whitney $U$ test; also Spearman's correlation analysis was done between IMT and age, anthropometric variables, systolic BP, diastolic BP, subcutaneous and visceral fat.

The project was accepted by the Local Committee of Research and Ethics in Research with the registration number R-2015-3606-22. All patients gave their consent to participate and signed the informed consent form.

\section{Results}

Eighty-six women were recruited and only 71 who met the inclusion criteria were analyzed.

Family history of cardiovascular disease in the first or second degree was present in 5 out 71, none had personal history of cardiovascular disease. Thirteen women were diabetic, 5 took glibenclamide plus metformin and other 5 used insulin. Four out of 71 have had breast cancer and 5 of 71 had smoking history. None of them had alcohol or drug consumption. Five out 71 did daily exercise, 9 weekly, 1 monthly and 56 were sedentary. Sixteen out of 71 considered their feeding habits as good, 1 regular and 54 bad.

The results are expressed in median and interval, in the whole group age was 55 (39-86) years, age at menarche 12 (10-17) years, age at menopause 50 (32-56) years; the number of pregnancies was 3 (0-12) and that of childbirths 2 (0-12). The height was $1.54(1.34-1.72) \mathrm{m}$, weight 66 (47-108) kg, BMI 27.60 (20.30-43.30), the waist perimeter $86(60-122) \mathrm{cm}$, the hip perimeter 99 (86-135) cm and the WHR 0.87 (0.69-1.05). Subcutaneous fat was $2.09(1.19-4.30) \mathrm{cm}$, visceral fat 4.60 $(1.70-14.80) \mathrm{cm}$ and the subcutaneous/visceral fat ratio 0.44 (0.16-1.28). The systolic BP was 120 (90-150) $\mathrm{mmHg}$ and the diastolic BP $70(60-90) \mathrm{mmHg}$. The carotid IMT was $0.60(0.10-1.90) \mathrm{mm}$.

A significant correlation was found between subcutaneous fat and BMI $(\rho=0.237, p<0.047)$, systolic BP correlated with the age $(\rho=0.234, p<0.051)$, the visceral fat $(\rho=0.250, p<0.036)$ and IMT $(\rho=0.282$, $p<0.017)$. The diastolic BP correlated with the age ( $\rho=0.238, p<0.047)$, IMT $(\rho=0.297, p<0.012)$ and IMT with the subcutaneous fat $(\rho=0.267, p<0.025)$.

After dividing the group according to abnormal group $1(n=9,12.7 \%)$ or normal group $2(n=62,87.3 \%)$ IMT, the waist perimeter, hip perimeter, subcutaneous 
Tab. I. Data in groups with increased and normal intima-media thickness

\begin{tabular}{|c|c|c|c|}
\hline Parameter & Group $1(n=9)$ & Group $2(n=62)$ & $p$ \\
\hline Age (years) & $58.6(52-73)$ & $56.2(39-86)$ & NS \\
\hline Menarche (years) & $12.56(10-16)$ & $12.73(10-17)$ & NS \\
\hline Menopause (years) & $47.78(36-53)$ & $48.60(32-56)$ & NS \\
\hline Pregnancies & $3(2-5)$ & $3.26(0-12)$ & NS \\
\hline Childbirth & $1.8(0-4)$ & $2.4(0-12)$ & NS \\
\hline Height (m) & $1.52(1.39-1.60)$ & $1.53(1.34-1.72)$ & NS \\
\hline Weight (kg) & $71.55(58-108)$ & $65.49(47-88)$ & NS \\
\hline Body mass index & $30.63(25.10-43.30)$ & $27.69(20.30-37.70)$ & NS \\
\hline Waist perimeter $(\mathrm{cm})$ & $95.11(84-122)$ & $85(60-105)$ & $<0.007$ \\
\hline Hip perimeter $(\mathrm{cm})$ & $110.33(92-135)$ & $98.38(86-114)$ & $<0.003$ \\
\hline Waist-hip ratio & $0.86(0.78-0.93)$ & $0.86(0.69-1.05)$ & NS \\
\hline Subcutaneous fat (cm) & $2.73(1.57-3.60)$ & $2.19(1.19-4.30)$ & $<0.018$ \\
\hline Visceral fat (cm) & $8.70(4.30-14.80)$ & $4.89(1.70-9.70)$ & $<0.001$ \\
\hline Systolic blood pressure $(\mathrm{mmHg})$ & $130(110-140)$ & $119.35(90-150)$ & $<0.006$ \\
\hline Diastolic blood pressure (mmHg) & $80(70-90)$ & $74.52(60-90)$ & NS \\
\hline Intima media thickness (mm) & $1.40(1.20-1.90)$ & $0.62(0.10-1.00)$ & $<0.001$ \\
\hline
\end{tabular}

Results are expressed in median and interval

Group 1: intima media thickness > $1 \mathrm{~mm}$. Group 2: intima media thickness $\leq 1 \mathrm{~mm}$

fat, visceral fat and systolic BP were significantly greater in group 1 (Table I).

In group 1 there was a correlation between $\mathrm{BMI}$ and subcutaneous fat ( $\rho=0.686, p<0.041$ ), between visceral fat and the systolic BP $(\rho=0.712, p<0.031)$, and in group 2 between IMT and the diastolic BP $(\rho=0.251$, $p<0.049)$.

\section{Discussion}

The atherosclerotic cardiovascular disease is more frequent after the menopause [2]. Increased IMT is associated with a higher risk of myocardial infarction and stroke [4]. In this study, it was found that women with increased IMT had significantly greater subcutaneous fat, visceral fat and systolic pressure. This indicates that the greater quantity of fat whether subcutaneous or visceral, increases the cardiovascular risk while increasing the carotid IMT which is an early marker of atherosclerosis, as already has been reported by other authors and in other populations [4, 7, 13, 14].

Also it was found that BP was significantly higher in those with greater carotid IMT, as has been reported by others who have demonstrated that hypertension, even though being subclinical is associated to atherosclerosis [15].

When correlating the variables in the group with increased IMT, visceral fat had a direct relationship with systolic BP, while in those with normal IMT, this correlated with the diastolic BP. All this confirms that visceral fat has an unfavorable role in cardiovascular health since higher BP is related with thicker IMT [15].

Although in this study no differences were found in WHR among the groups, the waist and hip perimeters were greater in those with thicker IMT, which also has been reported for the waist perimeter [16], and this waist perimeter has been associated with greater visceral fat.

This study had the following weaknesses since neither lipid levels nor diet details were investigated to settle down with more precision how these risk factors could increase the IMT, so protective measures can be established. Even though the small sample size after dividing the group according to abnormal or normal IMT, was enough to detect differences in waist and hip ratios, systolic blood pressure as well as in subcutaneous and visceral fat. But if samples size can be increased, maybe differences in BMI, WHR and diastolic blood pressure can be detected.

However, it is possible to conclude that subcutaneous fat and more the visceral fat have an unfavorable effect in the carotid IMT.

\section{Disclosure}

Authors report no conflict of interest.

\section{References}

1. Clinical practice guide. Diagnosis and treatment of perimenopause and postmenopause, Mexico Social Security Mexican Institute, 2013. (Guía 
de práctica clínica. Diagnóstico y tratamiento de la perimenopausia y postmenopausia. México. Instituto Mexicano del Seguro Social, 2013).

2. De Rosa J, Tonda A, Vita N. Postmenopausal status (Estado postmenopáusico). http://www.fac.org.ar/revista/99v28n4/derosa/derosa. htm.

3. Jarpa C, Pineda V, Manterola C, Grupo MINCIR. Carotid intima-media thickness as predictor of cardiovascular event. Systematic review of literature). Grosor de la íntima-media carotidea como predictor de evento cardiovascular. Revisión sistemática de la literatura. Int J Morphol 2013; 31: 293-300.

4. Coll B, Feinstein SB. Carotid intima-media thickness measurements: techniques and clinical relevance. Curr Atheroscler Rep 2008; 10: 444450.

5. The expert workgroup members. 2013 ACC/AHA Guideline on the Assessment of Cardiovascular Risk. J Am Coll Cardiol 2014; 63: 2935-2959.

6. Stein JH, Korcarz CE, Hurst RT, et al. Use of carotid ultrasound to identify subclinical vascular disease and evaluate cardiovascular disease risk: A consensus statement from the American Society of Echocardiography Carotid Intima-Media Thickness Task Force. Endorsed by the Society for Vascular Medicine. J Am Soc Echocardiogr 2008; 21: 93-111.

7. Lee MJ, Shin DH, Kim SJ, et al. Visceral fat thickness is associated with carotid atherosclerosis in peritoneal dialysis patients. Obesity (Silver Spring) 2012; 20: 1301-1307.

8. Porter S, Massaro JM, Hoffmann U, et al. Abdominal subcutaneous adipose tissue: A protective fat depot? Diabetes Care 2009; 32: 1068-1075.

9. Liu J, Fox CS, Hickson DA, et al. Impact of abdominal visceral and subcutaneous adipose tissue on cardiometabolic risk factors: the Jackson Heart Study. J Clin Endocrinol Metab 2010; 95: 5419-5426.

10. Pou KM, Massaro JM, Hoffmann U, et al. Visceral and subcutaneous adipose tissue volumes are cross-sectionally related to markers of inflammation and oxidative stress: the Framingham Heart Study. Circulation 2007; 116: 1234-1241.

11. De Lucia Rolfe E, Sleigh A, et al. Ultrasound measurements of visceral and subcutaneous abdominal thickness to predict abdominal adiposity among older men and women. Obesity (Silver Spring) 2010; 18: 625631.

12. Centers for Disease Control and Prevention EPI info 7.

13. Kollias A, Psilopatis I, Karagiaouri E, et al. Adiposity, blood pressure, and carotid intima-media thickness in Greek adolescents. Obesity 2013; 21: 1013-1017.

14. Ko GT, Chow CC, Leung G, et al. High rate of increased carotid intimamedia thickness and atherosclerotic plaques in Chineses asymptomatic subjects with central obesity. Int J Cardiovasc Imaging 2011; 27: 833841.

15. Cuspidi C, Sala C, Tadic M, et al. Untreated masked hypertension and carotid atherosclerosis: a meta-analysis. Blood Press 2015; 24: 65-71.

16. Asicioglu E, Kahveci A, Arikan $\mathrm{H}$, et al. Waist circumference is associated with carotid intima media thickness in peritoneal dialysis patients. Int Urol Nephrol 2013; 45: 1437-1443. 